\title{
Resonant spin tunneling in small antiferromagnetic particles
}

\author{
F. Luis \\ Instituto de Ciencia de Materiales de Aragon del Consejo Superior de Investigaciones Cientificas, \\ Universidad de Zaragoza, 5009 Zaragoza, Spain \\ E. del Barco and J. M. Hernández \\ Department de Física Fonamental, Universitat de Barcelona, Diagonal 647, 08028 Barcelona, Spain \\ E. Remiro and J. Bartolomé \\ Instituto de Ciencia de Materiales de Aragon del Consejo Superior de Investigaciones Cientificas, \\ Universidad de Zaragoza, 5009 Zaragoza, Spain \\ J. Tejada \\ Department de Física Fonamental, Universitat de Barcelona, Diagonal 647, 08028 Barcelona, Spain
}

(Received 17 June 1998; revised manuscript received 23 October 1998)

\begin{abstract}
The paper reports a detailed experimental study on magnetic relaxation of natural horse-spleen ferritin. ac susceptibility measurements performed on three samples of different concentration show that dipole-dipole interactions between uncompensated moments play no significant role. Furthermore, the distribution of relaxation times in these samples has been obtained from a scaling of experimental $\chi^{\prime \prime}$ data, obtained at different frequencies. The average uncompensated magnetic moment per protein is compatible with a disordered arrangement of atomic spins throughout the core, rather than with surface disorder. The observed field dependence of the blocking temperature suggests that magnetic relaxation is faster at zero field than at intermediate field values. This is confirmed by the fact that the magnetic viscosity peaks at zero field, too. Using the distribution of relaxation times obtained independently, we show that these results cannot be explained in terms of classical relaxation theory. The most plausible explanation of these results is the existence, near zero field, of resonant magnetic tunneling between magnetic states of opposite orientation, which are thermally populated.
\end{abstract}

[S0163-1829(99)08817-7]

\section{INTRODUCTION}

The study of quantum phenomena in materials having mesoscopic size magnetic units has been a fruitful research field for the past ten years. ${ }^{1}$ Recent experiments performed on samples of $\mathrm{Mn}_{12}$ acetate show that the nonequilibrium magnetization and the ac susceptibility approach abruptly their equilibrium limits at several values of the applied field. ${ }^{2-4}$ These field values fulfill resonance conditions, that is, some spin-up states are degenerate with some spin-down ones. Concurrently, experimentally obtained relaxation rates have sharp maxima at the same field values. ${ }^{2-6}$ These data give strong evidence for the existence of quantum resonant tunneling between degenerate spin states. Since it is found that the relaxation rates still follow Arrhenius' law at any value of the applied field, tunneling must take place between states which are thermally populated. Different theories have been proposed in order to account for the above-mentioned experiments. $^{7-9}$

The existence of resonant tunneling is directly related to the discreteness of the energy-level spectrum of a $\mathrm{Mn}_{12}$ acetate molecule. Therefore it seems reasonable to expect that similar effects can also occur in single domain particles having a limited amount of discrete energy levels. Particles made of antiferromagnetic materials are ideally suited for this purpose. The total spin of these particles is the result of a noncomplete compensation, due to finite-size effects of both magnetic sublattices. ${ }^{10,11}$ The total spin of an antiferromagnetic particle is therefore much smaller than that of a ferromagnetic particle of the same size. Energy differences between adjacent energy levels are correspondingly larger for the antiferromagnetic case. Thus these particles offer the possibility to observe resonant quantum phenomena at a larger size scale. Unfortunately, single domain particle systems are rather complex. Usually, assemblies of such particles have considerable size distribution and their easy axes of magnetization are randomly oriented. Therefore only at zero field the spin states of all particles are doubly degenerate. Another obstacle is that theoretical expressions for the measured quantities can only be obtained if the particles do not interact magnetically. A precise characterization of the magnetic behavior of a sample with various experimental techniques is therefore advisable.

The aim of this paper is to present a detailed experimental study of magnetic relaxation in natural horse-spleen ferritin. Ferritin is a protein which stores iron in mammals. ${ }^{12}$ It consists of a magnetic core of ferrihydrite surrounded by a protein shell. ${ }^{13}$ Each core is about $7 \mathrm{~nm}$ in diameter and can contain up to $4500 \mathrm{Fe}^{3+}$ ions. ${ }^{14}$ It is antiferromagnetic below $240 \mathrm{~K}$, but the protein may have a little uncompensated magnetic moment which arises from finite-size effects. It has been shown by different experimental techniques that ferritin behaves as an ideal superparamagnet above $20 \mathrm{~K}$, whereas magnetic moments become progressively frozen below that temperature. ${ }^{15-23}$ 
Awschalom et al. ${ }^{23}$ used ferritin in research on quantum tunneling. They observed a resonance, centered about 1 $\mathrm{MHz}$, in $\chi^{\prime \prime}(\omega)$ curves measured below $200 \mathrm{mK}$. They ascribed the resonance to the existence of coherent tunneling between degenerate spin states. Later, ferritin was also used in relaxation experiments of Ref. 16. It was observed that the magnetic viscosity, which measures the average relaxation rate of a sample, becomes temperature independent below $2.4 \mathrm{~K}$. This result could be seen as an experimental evidence of incoherent spin tunneling. ${ }^{16}$ Although both sets of experimental data point to the existence of quantum relaxation in ferritin, there is an apparent contradiction between them. On one hand, the resonance experiments suggest that most magnetic moments tunnel at frequencies of the order of $1 \mathrm{MHz}$ below $200 \mathrm{mK}$ whereas, on the other hand, relaxation experiments show that almost all the particles are already blocked at $4 \mathrm{~K}$ and observation times of $100 \mathrm{~s}$, approximately. Despite other experimental details, which mainly concern the sample preparation, the crucial difference is that the field applied to the sample in relaxation experiments is about six orders of magnitude larger than the ac field of Ref. 23. It is tempting to associate the slowing down of magnetic relaxation to the detuning, induced by the applied field, between the two lowest-lying energy states. ${ }^{24}$ This idea received experimental support recently, when Tejada and co-workers found that magnetic viscosity of natural ferritin increases markedly as the final field is reduced towards zero. ${ }^{20}$

In this paper, we give additional experimental evidence that resonant tunneling occurs in natural ferritin at zero field. It is shown that when the concentration of ferritin in the samples is increased by an order of magnitude, the ac susceptibility is not affected significantly, therefore magnetic interactions between different protein cores should play a minor role in explaining our experiments. The ac susceptibility provides independent information about the distribution of relaxation times and the average magnetic moment in our samples. Therefore, using this crucial information, we have been able to compare the experimental results with theoretical predictions for classical thermally activated relaxation that take into account the antiferromagnetic character of the particles. Our two main findings are (1) that the blocking temperature, obtained from dc and ac susceptibility experiments, increases as the applied field increases, and (2) that magnetic relaxation, either obtained from hysteresis measurements or from the magnetic viscosity, proceeds faster at zero field. Although, as already was pointed out in Refs. 22 and 25, an increase of the blocking temperature with the applied field can be explained classically as an effect due to the distribution of relaxation times, we observed that the experimental variation is faster than the classical prediction. Furthermore, the magnetic relaxation measured close to zero field clearly differs both quantitative and qualitatively, from the classical prediction that, on the other hand, explains reasonably well the data measured at higher fields. Thus we conclude that the combination of ac and dc susceptibility data and the magnetic viscosity cannot be understood in terms of classical relaxation, and needs the concourse of a magnetic quantum tunneling mechanism to be interpreted.

The paper is organized as follows. In the next section we give suitable expressions for the magnetic viscosity and ac susceptibility of a set of independent antiferromagnetic par- ticles. We extend previous calculations of the ac susceptibility to the case in which a nonzero magnetic field is applied to the sample. The experimental data are shown in Sec. III. Special care is taken of the characterization of the sample in the classical relaxation regime. In Sec. IV we show that our data are compatible with the existence of resonant tunneling at zero field. Finally, we list the main conclusions which follow from our work.

\section{THEORETICAL BACKGROUND}

We consider here a system of noninteracting single domain magnetic particles (SDP) dispersed in a nonmagnetic rigid matrix. We restrict ourselves to a case where magnetic anisotropy gives rise to a single preferred direction for the magnetic moment of each particle. Our aim here is to give expressions for the magnetic viscosity and ac susceptibility which can be compared to experiments, pointing out the different behavior of ferro- $(\mathrm{F})$ and antiferromagnetic (AF) particles. The magnetic moment $\mu$ of an AF particle arises from $N_{u c}$ uncompensated atomic moments $m$, that is, $\mu=N_{u c} m$. The magnitude of $N_{u c}$ depends on the type of atomic disorder which is at the origin of such lack of compensation. For simplicity, we assume that $N_{u c} \sim N^{p}$, where $N=\rho V, \rho$ is the density of atomic spins, $V$ is the particle's volume, and $p$ $\leqslant 1$. It has been proposed that $p=1 / 2$ if disorder extends through the whole particle, ${ }^{10}$ whereas $p=1 / 3$ if it is restricted to the surface. ${ }^{11}$

\section{A. Magnetic viscosity}

Let us first consider a set of SDP of volume $V$ whose easy axes are aligned and make an angle $\psi$ with respect to the applied field $H$. The relaxation rate $\Gamma$ for the evolution towards the equilibrium magnetization is given by

$$
\Gamma=\Gamma_{0} \exp \left[-\frac{U}{k_{B} T^{*}}\right] .
$$

Here $\Gamma_{0}$ is an attempt frequency, $U$ is an activation energy, and $T^{*}$ is an effective temperature which depends on the relaxation mechanism. $T^{*}$ equals $T$ if relaxation takes place by thermally activated transitions over the energy barrier, whereas it becomes equal to a constant $T_{Q}$, that depends only weakly on $V$, if quantum tunneling between lowestlying states is most probable. The crossover between both regimes occurs at $T=T_{Q}$. In both cases, assuming uniform rotation of all atomic spins in the particle and that the field makes an angle $\psi$ with respect to the easy axes, $U$ $=K_{0} V g(V, H, \psi)$, where $K_{0}$ is the density of anisotropy energy and $g$ is given by ${ }^{26}$

$$
g(V, H, \psi) \simeq\left[1-H / H_{a}(\psi)\right]^{\kappa(\psi)} .
$$

Here, $H_{a}(\psi) \simeq H_{a}(0)\left[(\sin \psi)^{2 / 3}+(\cos \psi)^{2 / 3}\right]^{-3 / 2}$ is the anisotropy field, and $\kappa \simeq 0.86+1.14\left[H_{a}(\psi) / H_{a}(0)\right]$. A few words about the physical meaning of $H_{a}(0)$ may be appropriate here. Equation (2) is only valid at low enough fields such that the magnitude of $\mu$ does not differ appreciably from its zero-field value. In this field range $H_{a}(0)=2 K_{0} V / \mu$. Consequently, $H_{a}$ is independent of $V$ only if $\mu$ is proportional to it, which is not the case for an AF particle. At higher fields a 
decrease of the effective anisotropy field is expected, due to the field induced increase of $\mu$. However, this effect can be relatively small if the exchange interaction between the atomic spins is large enough. It follows that the anisotropy field of a single domain AF particle can be much larger (by a factor of $N / N_{u c}$, approximately) than it is for each magnetic sublattice of the bulk material.

For a sample containing particles of different size, the magnetization $M$ of the sample is a superposition of contributions, each decaying exponentially in time towards its equilibrium value, with a different $\Gamma$ given by Eq. (1). Since magnetization decay is measured during several hours and $\Gamma_{0} \sim 10^{9}-10^{13} \mathrm{~s}$, the $\exp (-\Gamma t)$ factor is essentially the theta function of $U-U_{b}$, where $U_{b}(t)=T^{*} \ln \left(\Gamma_{0} t\right)$ is the activation energy of the particles that contribute most to the magnetic relaxation. If the easy axes of all particles are aligned it is possible to calculate the evolution of $M$ with time as ${ }^{27}$

$$
M(t)=M_{e q}(H, T)+\int_{V_{b}}^{\infty}\left[M_{0}(V)-M_{e q}(V, H, T)\right] f(V) d V .
$$

Here $f(V)$ is the fraction of total sample's volume that is occupied by particles of volume $V$ and $V_{b}$ fulfills $K_{0} V_{b} g$ $=U_{b}$. We assume that $f(V)$ is a smooth function of $V$, as it is indeed the case for ferritin (see below). Note that $M_{e q}(H, T)$ is the equilibrium magnetization of the whole sample at the field $H$ whereas, in contrast, $M_{e q}(V, H, T)$ refers only to a subset of particles having a given size. $M_{0}(V)$ is the magnetization of this subset at $t=0$, after all reversible magnetization processes have died out.

If the particles' easy axes are oriented at random, the interaction with an applied field can broaden the distribution of energy barriers with respect to the distribution of volumes. ${ }^{22,26,28,29}$ Equation (3) can still be used provided the applied field is small enough in comparison to the anisotropy field $H_{a}$. In this case, $\left[M_{0}(V)-M_{e q}(V, H, T)\right]$ must be averaged over all particle orientations.

The time derivative of $M$ is given by

$$
\frac{\partial M}{\partial \ln (t)}=-\frac{k_{B} T^{*}}{K_{0} g(V, H, \psi)} f\left(V_{b}\right)\left[M_{0}\left(V_{b}\right)-M_{e q}\left(V_{b}, H\right)\right] .
$$

Here, both $V_{b}$ and $\left[M_{0}\left(V_{b}\right)-M_{e q}\left(V_{b}, H\right)\right]$ depend on temperature and field. We note, as another important feature distinctive to $\mathrm{AF}$ particles, that the saturation magnetization $M_{s} \propto N_{u c} / N$, therefore it depends on the particles' volume. Accordingly, $\left[M_{0}\left(V_{b}\right)-M_{e q}\left(V_{b}, H\right)\right]$ depends on temperature for almost any experimental condition. It is therefore convenient to estimate experimentally this temperature dependence before we extract from $[\partial M / \partial \ln (t)]$ any information concerning the nature (quantum or classical) of the relaxation process. A more convenient definition of magnetic viscosity follows:

$$
S(T, H)=-\frac{1}{\left[M_{0}\left(V_{b}\right)-M_{e q}\left(V_{b}, H\right)\right]} \frac{\partial M}{\partial \ln (t)},
$$

which, according to Eq. (4), is independent of equilibrium magnitudes.

\section{B. ac susceptibility at zero field}

The zero-field ac magnetic susceptibility $\chi$ of noninteracting ferromagnetic particles has been calculated elsewhere. ${ }^{30}$ Here we will only rewrite the expressions given in Ref. 30 in order to take into account the antiferromagnetic character of ferritin. We also consider that the easy axes of magnetization are randomly oriented. Using simple approximations ${ }^{30,31}$ the following expressions apply:

$$
\chi^{\prime} \simeq \int_{0}^{V_{b}} \chi_{e q}(V, T) f(V) d V+\frac{2}{3} \int_{V_{b}}^{\infty} \chi_{\perp}(V, T) f(V) d V+\chi_{a f},
$$

$$
\chi^{\prime \prime} \simeq \frac{\pi}{6 K_{0}} k_{B} T^{*} \chi_{e q}\left(T, V_{b}\right) f\left(V_{b}\right)
$$

where $V_{b}(\omega)$ now simply equals $U_{b} / K_{0}, \chi_{e q}=\frac{2}{3} \chi_{\perp}+\frac{1}{3} \chi_{\|}$, $\chi_{\|}, \chi_{\perp}$ are the equilibrium zero-field susceptibilities along directions parallel and perpendicular to the anisotropy axis, ${ }^{30}$ respectively, and $\chi_{a f}$ is the antiferromagnetic susceptibility. As explained in Ref. 30, the response to the component of the applied field which is parallel to the easy axis involves overcoming the energy barrier and it leads to irreversibility when $V>V_{b}$. In contrast, for $\omega \Gamma_{0} \ll 1$, the response to a field perpendicular to the easy axis is fully reversible. The first two terms in Eq. (6) give the contribution of the uncompensated moments.

If $K_{0} V \gg k_{B} T$ then $\chi_{e q} \simeq \mu^{2} / 3 V k_{B} T$. Thus, according to Eq. (7), if all magnetic moments reverse by a thermally activated relaxation process, $\chi^{\prime \prime}$ is a function only of $V_{b}$ because $K_{0} V_{b} \gg k_{B} T$. Since the value of $V_{b}$ for a given temperature depends on $\Gamma_{0}$, best superposition of experimental $\chi^{\prime \prime}(T)$ curves measured at different frequencies gives an estimation of this parameter. Fitting experimental data to Eq. (7), the distribution of volumes in a given sample can be obtained. This experimental technique was first applied to obtain the fraction of particles of a $\mathrm{CrO}_{2}$ recording tape which are superparamagnetic at room temperature. ${ }^{32}$

From Eqs. (6) and (7) it follows that $\chi^{\prime}$ and $\chi^{\prime \prime}$ have a maximum centered about $T_{B}\left(\chi^{\prime}\right)$ and $T_{B}\left(\chi^{\prime \prime}\right)$, respectively. It can also be shown that, within a good approximation, these maxima shift with $\omega$ according to

$$
\ln \left(\frac{\Gamma_{0}}{\omega_{0}}\right)=\frac{U_{e f}}{k_{B} T_{B}} .
$$

We shall denote by $U_{e f}^{\prime}$ and $U_{e f}^{\prime \prime}$ the effective energies for $\chi^{\prime}$ and $\chi^{\prime \prime}$, respectively. They are, respectively, solutions of

$$
U_{e f}^{\prime}=\frac{\int_{0}^{U_{e f}^{\prime}}\left(\mu^{2} / U\right) f\left(U / K_{0}\right) d U}{\left\{\left[\mu\left(U_{e f}^{\prime}\right)\right]^{2} / U_{e f}^{\prime}\right\} f\left(U_{e f}^{\prime}\right)}
$$

and

$$
(2 p-1) f\left(U_{e f}^{\prime \prime}\right)+\left.U_{e f}^{\prime \prime} \frac{\partial f}{\partial U}\right|_{U=U_{e f}^{\prime \prime}}=0,
$$

which follow from a straightforward derivation of Eqs. (6) and (7). We emphasize that, in general, neither $U_{e f}^{\prime}$ nor $U_{e f}^{\prime \prime}$ 


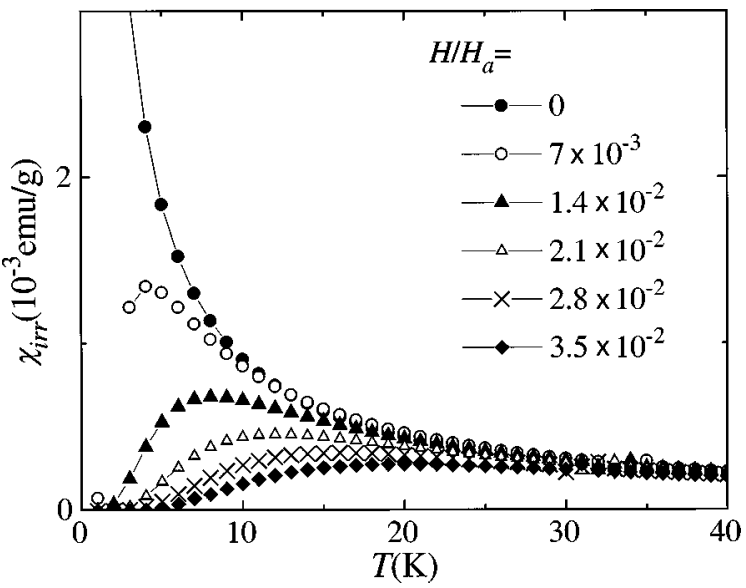

FIG. 1. Irreversible component of the equilibrium susceptibility of a set of noninteracting identical SDP calculated for $\psi=\pi / 4$ and different values of the applied field in units of the maximum anisotropy field (these values correspond, respectively, to $H=0,0.2,0.4$, $0.6,0.8,1 \mathrm{kOe}$ for ferritin). The parameters $K_{0} V=324 k_{B}, K_{0}$ $=2.5 \times 10^{5} \mathrm{erg} / \mathrm{cm}^{3}$, and $\mu=334 \mu_{B}$, which correspond to the average values for ferritin, were used in the calculations. The concentration of magnetic particles is the same as in sample $\mathrm{S}$.

equal the average energy barrier $K_{0}\langle V\rangle$ of a given sample. Instead, they can depend strongly on the width of the volumes' distribution $f$.

If, in contrast, magnetic moments tunnel between lowestlying states, localized on opposite sides of the anisotropy energy barrier, $V_{b}$ becomes temperature independent. In that case, those magnetic moments which are superparamagnetic at $T=T_{Q}$ remain in thermal equilibrium down to $0 \mathrm{~K}$. These particles give therefore the most important contribution to $\chi^{\prime}$ below $T_{Q}$. Moreover, $\chi^{\prime \prime} \simeq\left(T_{Q} / T\right) \chi^{\prime \prime}\left(T_{Q}\right)$ [see Eq. (7)]. Thus, irrespective of the details of $f(V)$ and of the equilibrium susceptibilities, $\chi^{\prime}$ and $\chi^{\prime \prime}$ must increase as 1/T below $T_{Q}$.

\section{C. ac susceptibility under an applied dc magnetic field}

A magnetic field destroys the symmetry around the easy axis of each particle. Therefore reversible $\chi_{\text {rev }}$ and irreversible $\chi_{i r r}$ responses to the ac field are no longer given by parallel and perpendicular susceptibilities, respectively.

Let us denote by $\theta_{\min }^{(1)}$ and $\theta_{\min }^{(2)}$, the position of the two energy minima of a given SDP. $\chi_{\text {rev }}$ appears as a result of the small variation, induced by the ac field, of $\theta_{\min }^{(1)}$ and $\theta_{\min }^{(2)}$, whereas the change of their relative equilibrium populations gives rise to $\chi_{i r r}$. At low temperatures, when occupation probabilities of excited states within each anisotropy energy well are very small, we can roughly estimate $\chi_{r e v}$ as follows:

$$
\chi_{\text {rev }} \simeq \frac{\mu}{V}\left(P_{1} \frac{\partial \cos \left(\psi-\theta_{\text {min }}^{(1)}\right)}{\partial H}+P_{2} \frac{\partial \cos \left(\theta_{\min }^{(2)}-\psi\right)}{\partial H}\right),
$$

where $\quad P_{i}=(1 / Z) \exp \left\{-\left[E\left(\theta_{\text {min }}^{(i)}\right)-E\left(\theta_{\text {min }}^{(1)}\right)\right] / k_{B} T\right\}, \quad Z=1$ $+\exp \left\{-\left[E\left(\theta_{\min }^{(2)}\right)-E\left(\theta_{\min }^{(1)}\right)\right] / k_{B} T\right\}$, and $\psi$ is the angle of the applied field with respect to the easy axis. Using this expression $\chi_{i r r}$ can be estimated as $\chi_{e q}-\chi_{r e v}$, since it is relatively easy to calculate numerically $\chi_{e q} \cdot{ }^{33}$ We have plotted in Fig. $1 \chi_{i r r}$ as a function of the temperature for several values of the applied field and $\psi=\pi / 4$. We see that the magnitude of $\chi_{i r r}$ decreases as $H$ increases whereas $\chi_{\text {rev }}$ (not shown) was found to depend weakly on the applied field and temperature. There is still another important feature of Fig. 1 which must be considered. Even at relatively low fields, $\chi_{i r}$ deviates significantly from the $1 / T$ dependence. Moreover, it has a peak at a finite temperature which increases as the field increases. From this calculation it follows that, even for pure classical relaxation, the values of $T_{B}\left(\chi^{\prime}\right)$ and $T_{B}\left(\chi^{\prime \prime}\right)$ not only depend on the relaxation times of the particles, but also on equilibrium properties. Thus they can either increase or decrease as $H$ increases.

In order to compare numerical calculations of the ac susceptibility to our experimental results for ferritin it is still necessary to introduce a distribution of particles' volumes and orientations. We consider, as we did at zero field, that the response of blocked particles (which fulfill $\omega / \Gamma>1$ ) is just equal to $\chi_{\text {rev }}$ (because reversible processes take place in time intervals of the order of $1 / \Gamma_{0} \sim 10^{-12} \mathrm{~s}$, much shorter than the time scale $10^{-3}<1 / \omega<10^{-1}$ s of our ac measurements) and that superparamagnetic particles (for which $\omega / \Gamma<1)$ are in equilibrium. Similarly, we only consider contributions to $\chi^{\prime \prime}$ of those particles which fulfill $\omega / \Gamma=1$. Using these approximations, it follows that

$$
\begin{aligned}
\chi^{\prime} \simeq & \chi_{r e v}+\int_{0}^{\pi / 2}\left(\int_{0}^{U_{b}} \chi_{i r r}(U, T, H, \psi) f[V(U, H, \psi)] \frac{\partial V}{\partial U} d U\right) \\
& \times \sin \psi d \psi, \\
\chi^{\prime \prime} & \simeq \frac{\pi k_{B} T^{*}}{2 K_{0}} \int_{0}^{\pi / 2} \frac{\chi_{i r r}\left(V_{b}, T, H, \psi\right) f\left(V_{b}\right)}{g+\kappa(1-p)\left(1-g^{1 / \kappa}\right)(g)^{1-1 / \kappa}} \sin \psi d \psi,
\end{aligned}
$$

where $g$ has been defined in Eq. (2).

Equations (12) and (13) are, in fact, direct generalizations of Eqs. (6) and (7), respectively. For thermally activated relaxation, it follows that $\chi^{\prime}$ tends to $\chi_{\text {rev }}$, which we found to be weakly dependent on the applied field, as $T$ goes to 0 . In contrast, since the irreversible contribution of the superparamagnetic particles to the susceptibility decreases as $H$ increases, both $\chi^{\prime}$ and $\chi^{\prime \prime}$ must decrease too at finite temperatures. Below, we shall use these theoretical developments to interpret our experimental results.

\section{EXPERIMENT}

\section{A. Experimental details}

The samples used in these experiments were obtained by varying the concentration of commercial ferritin from horse spleen (Fluka Biochemika, 46230). Sample D (diluted) is just the commercial one. Samples C (concentrated) and S (solid) were obtained from this one by reducing the amount of solvent using two different methods. For sample $\mathrm{C}$, the original diluted solution was mixed with $\mathrm{K}_{2} \mathrm{SiO}_{4}$ and then centrifuged for about one hour. Sample $\mathrm{S}$ was obtained using a microconcentrator MICROSEP, which has a cutoff mass at $30 \mathrm{KD}$. Magnetic measurements were performed in a Quantum Design superconducting quantum interference device magnetometer with the ac susceptibility measurement option. 


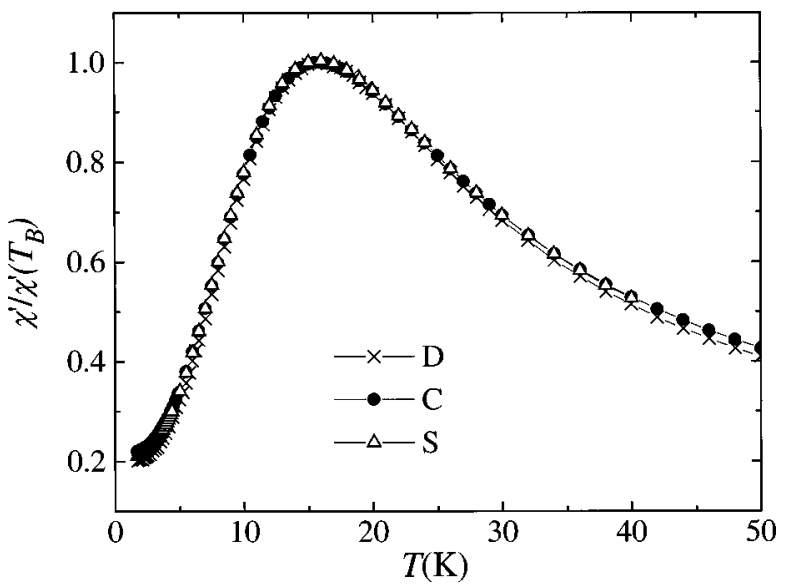

FIG. 2. Comparison between $\chi^{\prime}(T)$ measured at $\nu=9 \mathrm{~Hz}$ for the three samples investigated in this work. Susceptibility curves have been normalized to their respective maximum values.

The sample holder for sample D was made of quartz, and its magnetic signal was undetectable. For samples $\mathrm{C}$ and $\mathrm{S}$, the diamagnetic signal of the sample holder was, in any case, negligible with respect to that of the sample. Hysteresis cycles and ac susceptibility were measured after cooling the sample at zero applied field. Magnetic relaxation experiments were performed as follows: first the sample was cooled in zero field from the superparamagnetic state down to the measurement temperature, then the field was applied and we observed the ensuing evolution of the magnetization to its equilibrium state, determined by the field and temperature.

\section{B. Sample characterization in the thermally activated relaxation regime}

Dipolar magnetic interactions between different particles can modify the height of energy barriers separating different orientations of a magnetic moment. It is therefore desirable to obtain samples where such interaction effects may safely be neglected. We next show that it is indeed the case for natural ferritin. Experimental $\chi^{\prime}$ data, normalized to their respective maximum values, for samples $\mathrm{D}, \mathrm{C}$, and $\mathrm{S}$ are shown in Fig. 2. The ratio between normalization factors for samples D, C, and S is 1:5:17, from which we directly obtain the ratio between their concentrations. These curves neatly superimpose one on each other. Thus the magnetic response and relaxation times of the particles are not affected as we increase the concentration by more than an order of magnitude. Therefore our experimental data give the magnetic response of a set of independent magnetic particles. This agrees with the fact that the zero-field cooled magnetization data follow Curie's law above the blocking temperature.

In-phase $\chi^{\prime}$ and out-of-phase $\chi^{\prime \prime}$ components of the magnetic susceptibility for sample D are plotted in Fig. 3 as a function of temperature. Above $30 \mathrm{~K}, \chi^{\prime}$ follows approximately Curie's law and, correspondingly, $\chi^{\prime \prime} \simeq 0$. That is, magnetic moments are superparamagnetic in the time scale of our experiments. Below $30 \mathrm{~K}$, the blocking of progressively smaller magnetic moments leads to a decrease of $\chi^{\prime}$ and to the onset of a nonzero $\chi^{\prime \prime}$ value. $T_{B}\left(\chi^{\prime}\right)$ and $T_{B}\left(\chi^{\prime \prime}\right)$ increase as the frequency $\omega / 2 \pi$ of the applied field increases.

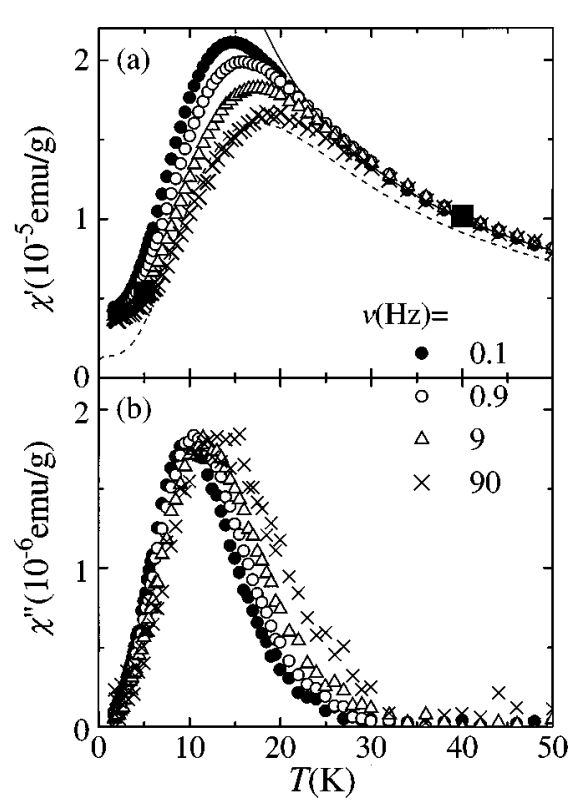

FIG. 3. Frequency dependence of the ac susceptibility of sample D. (a) In-phase component; the continuous line is a least-squares fitting to Curie's law of data measured above $30 \mathrm{~K}$; the dotted line represents the contribution due to uncompensated spins for $\nu$ $=9 \mathrm{~Hz}$ calculated numerically with Eq. (6) and the parameters of Table II. At $T=5 \mathrm{~K}$ and $T=40 \mathrm{~K}$ the antiferromagnetic contribution, estimated from magnetization measurements, has been added to the calculated values (large square dots). (b) Imaginary component.

This frequency dependence can be fitted to Eq. (8) in order to estimate $\Gamma_{0}, U_{e f}^{\prime}$ and $U_{e f}^{\prime \prime}$. Fitting parameters obtained in this way for samples D, C, and S are listed in Table I. We note that $U_{e f}^{\prime}$ and $U_{e f}^{\prime \prime}$ are clearly different from each other, which results from the finite width of $f(V)$.

Since dipolar interactions are negligible we can obtain $f(V)$ [actually we obtain $f\left(K_{0} V\right)$ ] in our sample from $\chi^{\prime \prime}$ experimental data making use of Eq. (7). Indeed, as predicted in the previous section, $\chi^{\prime \prime}(T)$ data measured at different frequencies merge into a single curve when represented as a function of the scaling variable $K_{0} V_{b}$ $=k_{B} T \ln \left(\Gamma_{0} / \omega\right)$ for $\Gamma_{0} \simeq 10^{12} \mathrm{~s}^{-1}$ (see Fig. 4). This value for $\Gamma_{0}$ agrees reasonably well with that obtained fitting the shift of $T_{B}\left(\chi^{\prime}\right)$ and $T_{B}\left(\chi^{\prime \prime}\right)$ with frequency to Eq. (8), and with previous estimations obtained from different experimental techniques. ${ }^{15,18}$ It is advisable to have an analytical expression of $f(V)$ which can be used to calculate other physical properties. We have tried to fit our experimental data using a

TABLE I. Parameters obtained by fitting the frequency dependence of $T_{B}\left(\chi^{\prime}\right)$ and $T_{B}\left(\chi^{\prime \prime}\right)$ to Eq. (8) for the three samples investigated. Calculated values follow from Eqs. (9) and (10) using the distribution of volumes obtained experimentally (see below).

\begin{tabular}{lccc}
\hline \hline Sample & $U_{e f}^{\prime} / k_{B}(\mathrm{~K})$ & $U_{e f}^{\prime \prime} / k_{B}(\mathrm{~K})$ & $\Gamma_{0}\left(\mathrm{~s}^{-1}\right)$ \\
\hline $\mathrm{D}$ & $418(50)$ & $269(70)$ & $1 \times 10^{12(2)}$ \\
$\mathrm{C}$ & $409(50)$ & $272(20)$ & $1 \times 10^{12(1)}$ \\
$\mathrm{S}$ & $396(50)$ & $267(25)$ & $2 \times 10^{12(1)}$ \\
Calculated & 417 & 270 & \\
\hline \hline
\end{tabular}




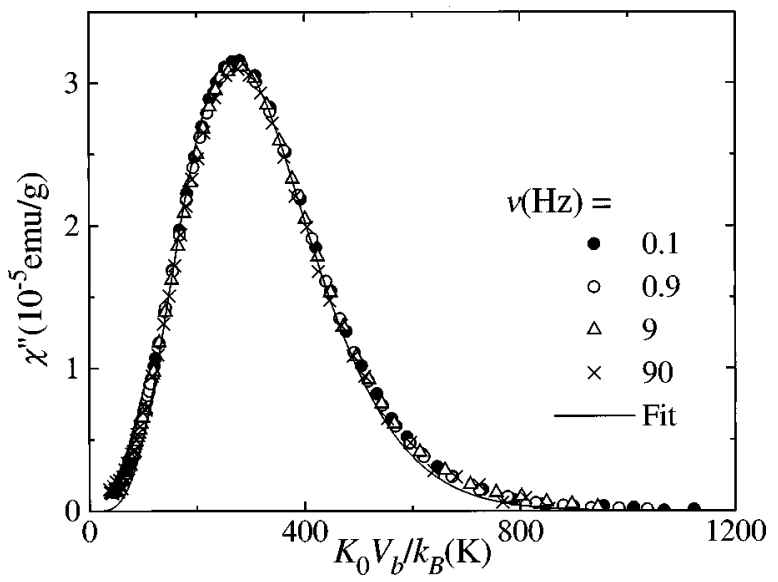

FIG. 4. Plot of $\chi^{\prime \prime}$ for sample $S$ as a function of the scaling variable $K_{0} V_{b} / k_{B}$ for $\Gamma_{0}=10^{12} \mathrm{~s}^{-1}$. The continuous line is the best fit to Eq. (7) for $p=1 / 2$ using a gamma function [Eq. (14)] to describe the distribution of particles' volumes. Fitting parameters are given in Table II.

$\log$ normal, a Gaussian, and a gamma function for $f(V)$. The best fit has been obtained using a gamma function (see Fig. 4):

$$
f\left(K_{0} V\right)=\frac{x}{U_{\Gamma}}\left(\frac{K_{0} V}{U_{\Gamma}}\right)^{\beta} \frac{\exp \left(-K_{0} V / U_{\Gamma}\right)}{\Gamma(\beta+1)},
$$

where $x$ is the magnetic fraction of the sample. Since, in our case, only the total mass, and not the volume, of the sample is measured, $x$ is given in terms of volume of magnetic cores per unit mass of the sample. The parameters $U_{\Gamma}$ and $\beta$ are listed in Table II for $p=1 / 2$ and $p=1 / 3$. It follows from Eqs. (9) and (10) that $U_{e f}^{\prime \prime}=(\beta+2 p-1) U_{\Gamma}$ and that $U_{e f}^{\prime}$ $=\zeta U_{\Gamma}$, where $\zeta$ is a solution of $y(\zeta)=\int_{0}^{\zeta} y(z) d z$ and $y(z)$ $=z^{2 p+\beta-1} \exp (-z)$. Calculated values for these two parameters are compared to experimental ones in Table I. The agreement is satisfying.

Once the distribution function is known, numerical integration of Eq. (7) gives directly $\chi^{\prime}$. The result of this calculation (dashed line) is compared to the experimental results in Fig. 3(a). As shown in the figure, the difference between experimental data and the calculated curve at $T=5$ and $40 \mathrm{~K}$ equals approximately $\chi_{a f}$ that was estimated from the highfield slope of magnetization curves (see below). We found that $\chi_{a f}$, estimated either from dc and ac experimental data, decreases as the temperature increases, as pointed out recently by other authors. ${ }^{19}$ Néel associated this behavior to the decrease of the polarizability of surface magnetic moments. ${ }^{10}$

Independent experimental results can be useful to estimate which value of $p$ is most appropriate to ferritin, that is, to determine the atomic spin arrangement in the protein cores. The saturation magnetization $M_{s}$ of a sample of antiferromagnetic particles is approximately equal to

TABLE II. Parameters of the distribution of particles' volumes obtained by fitting the experimental data of Fig. 4 to Eq. (7).

\begin{tabular}{lccc}
\hline \hline$p$ & $\pi x K_{0} m^{2}\left(\rho k_{B} / K_{0}\right)^{2 p} / 6 k_{B}^{2}\left(\mathrm{emu} \mathrm{K} \mathrm{K}^{2(1-p)} / \mathrm{g} \mathrm{Oe}\right)$ & $\beta$ & $U_{\Gamma} / k_{B}(\mathrm{~K})$ \\
\hline $1 / 2$ & $9.77(6) \times 10^{-3}$ & 5 & $54.0(2)$ \\
$1 / 3$ & $6.71(3) \times 10^{-2}$ & 5 & $57.8(1)$ \\
\hline \hline
\end{tabular}

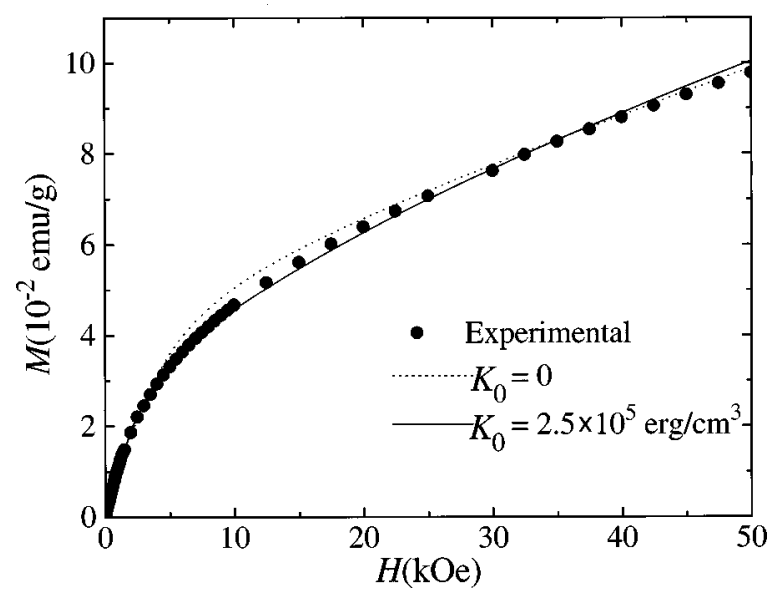

FIG. 5. Isothermal magnetization curve for sample $\mathrm{D}$ at $T$ $=40 \mathrm{~K}$. Results of numerical calculations for two different values of the anisotropy energy density $K_{0}$ are also shown. The parameters of the calculation are those of Table II $(p=1 / 2)$ and $\rho=2.5$ $\times 10^{22}$ ions $/ \mathrm{cm}^{3}$, and $\chi_{a f}=10^{-6} \mathrm{emu} / \mathrm{g}$.

$x m \rho^{p} V^{p-1}$, being therefore sensitive to the fraction of uncompensated atomic moments. Moreover, it is relatively easy to calculate the equilibrium $M(H)$ magnetization curve at a given temperature. ${ }^{33}$ We have used the parameters obtained from the susceptibility, which are given in Table II, and taken $\rho \simeq 2.5 \times 10^{22}$ ions $/ \mathrm{cm}^{3}, \quad m=5 \mu_{B}$ and $K_{0} \simeq 2.5$ $\times 10^{5} \mathrm{erg} / \mathrm{cm}^{3}$ from the literature. ${ }^{12,20}$ The antiferromagnetic contribution $\chi_{a f} H$ has been estimated from the high-field slope of the experimental magnetization curves (which, for $H>20 \mathrm{kOe}$ gives $\chi_{a f} \simeq 10^{-6} \mathrm{emu} / \mathrm{g}$ for sample $\mathrm{D}$ at $T$ $=40 \mathrm{~K}$ ). A good agreement with experimental data for $40 \mathrm{~K}$ is obtained for $p=1 / 2$ (see Fig. 5), whereas $p=1 / 3$ led to a completely wrong result. That is, only $p=1 / 2$ is compatible with both the temperature dependence of $\chi^{\prime \prime}$ and the value of $M_{s}$. Thus it seems that not only surface atoms deviate from the perfect antiferromagnetic order in natural ferritin.

We have also observed that magnetic anisotropy does not modify drastically the equilibrium magnetization curves of ferritin samples (the curve calculated for $K_{0}=0$ is only slightly above the experimental data at intermediate field values). It explains that, within the experimental errors, equilibrium $M(H / T)$ data measured between 10 and $100 \mathrm{~K}$ scale on a single curve. ${ }^{20}$

From $K_{0}\langle V\rangle \simeq 324 k_{B}$ and $K_{0} \simeq 2.5 \times 10^{5} \mathrm{erg} / \mathrm{cm}^{3}$, which have been just determined, it follows that the average diameter of ferritin cores is about $7 \mathrm{~nm}$. This value is nearly identical to the one obtained from previous TEM experiments. $^{12}$ The average net magnetic moment is then $334 \mu_{B}$ per protein core.

\section{Effect of the applied field on the magnetic susceptibility}

We have measured the temperature and frequency dependence of $\chi^{\prime}$ and $\chi^{\prime \prime}$ under a dc applied field. Experimental results for $\omega / 2 \pi=9 \mathrm{~Hz}$ are shown in Fig. 6. The maximum value of both components decreases as the applied field increases, whereas the low-temperature limit of $\chi^{\prime}$ is nearly field independent. As we discussed at the end of Sec. II, this field dependence can be qualitatively explained taking into account the different effect that the applied field has on the 


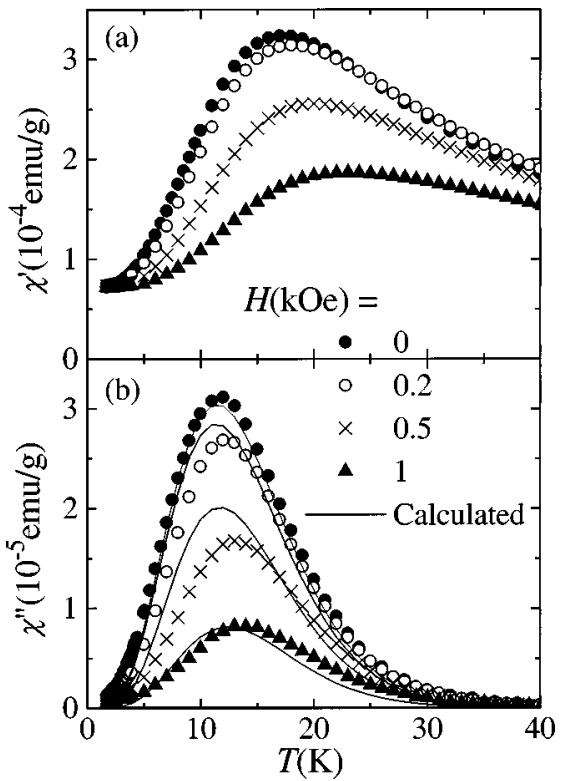

FIG. 6. Ac susceptibility of sample S measured at $\nu=9 \mathrm{~Hz}$ and four values of the applied field. (a) In-phase component; (b) out-ofphase component. Continuous lines in (b) represent the results of numerical calculations of Eq. (13) at the same fields, performed with the parameters of Table II.

irreversible and reversible equilibrium susceptibilities (see also Fig. 1). Thus, only a quantitative comparison between the classical predictions of Sec. II and the experimental results can help to infer how the applied field modifies magnetic relaxation.

We calculated numerically $\chi^{\prime \prime}$ with Eq. (13). The volume distribution that we have previously obtained at zero field was used in the calculation. The numerical results are compared to the experimental data in Fig. 6(b). Although the theoretical curves reproduce the main features of the experiments, there are some quantitative differences between them. First, the measured susceptibility decreases faster at intermediate fields and, second, $T_{B}\left(\chi^{\prime \prime}\right)$ shifts with field appreciably more than it is predicted classically. This is more clearly seen in Fig. 7. We emphasize that Eq. (13), that we used to calculate the theoretical curves, has been obtained assuming that the uncompensated moments do not change appreciably with the applied field. As discussed in Sec. II, the field induced increase of $\mu$ tends to decrease the value of the anisotropy field, thus increasing the effect of the applied field on the energy barriers. Therefore this effect would even enlarge the difference between the experimental data and the classical predictions.

In Fig. 7 we also compare the classical predictions with the blocking temperature $T_{B}$ obtained previously from zerofield cooled dc susceptibility, that presents a minimum at zero field too. ${ }^{20-22}$ In the calculations, we followed the method described in Refs. 25 and 34 but making $\mu \propto V^{1 / 2}$ that, as we have shown just before, is appropriate for ferritin. Furthermore, we consider a random orientation of the easy axes and, as for the ac case, we made use of the distribution of volumes obtained independently for the same sample (see Table II). The time was taken equal to $1 \mathrm{~min}$. The calculations show that $T_{B}$ increases with $H$, as suggested in Refs. 25 and 34, when nonlinear effects become important (above 0.5

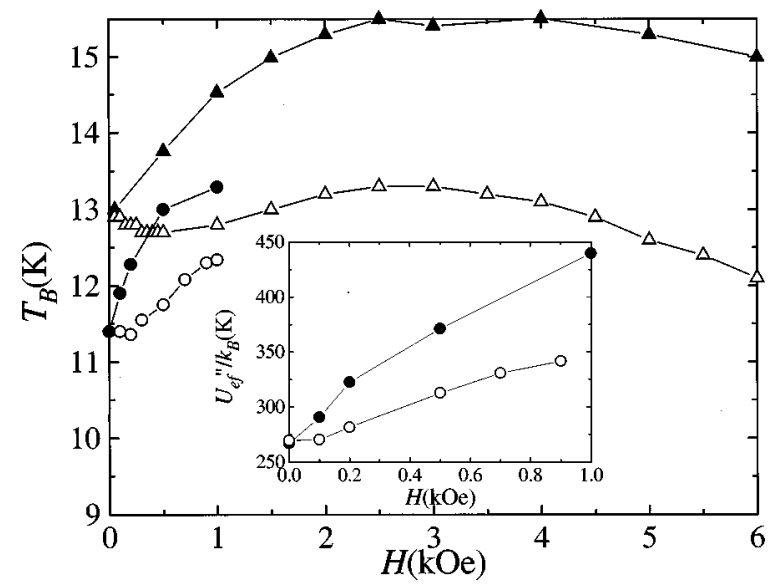

FIG. 7. Field dependence of the blocking temperature obtained from the data of Fig. 6(b) (dots) and from dc susceptibility experiments (triangles). The field dependence of $U_{e f}^{\prime \prime}$ is shown in the inset. The open symbols represent the classical predictions including the effect of the distribution of sizes (see text).

kOe in our numerical calculations), reaches a maximum and decreases at higher fields, in qualitative agreement with the experimental data. We note that $T_{B}$ obtained from ac or dc susceptibility experiments can increase with field even for a classical relaxation mechanism. Thus definite conclusions about the existence or not of resonant tunneling cannot be drawn just from the sign of $\partial T_{B} / \partial H$ near $H=0$. However, both the slope at low field (as in the calculations of Hanson, Johansson, and Morup ${ }^{25}$ ) and the increase between $H=0$ and $3 \mathrm{kOe}(1 \mathrm{kOe}$ for the ac data) are much smaller than what is observed experimentally.

We have observed that the frequency dependence of $T_{B}\left(\chi^{\prime}\right)$ and $T_{B}\left(\chi^{\prime \prime}\right)$ obtained experimentally can be fitted reasonably well to Arrhenius' law. The corresponding energy barriers $U_{e f}^{\prime}$ and $U_{e f}^{\prime \prime}$ have minima at zero field too. The inset of Fig. 7 shows, as a function of the applied field, $U_{e f}^{\prime \prime}$ data obtained experimentally and those deduced from numerical calculations for classical relaxation. It is clear again that, although both sets of data present a minimum at zero field, the variation of $U_{e f}^{\prime \prime}$ that we observed experimentally between $H=0$ and $H=1 \mathrm{kOe}$ is appreciably larger than what follows from a classical model.

Briefly, the above data clearly indicate that, in contrast to the predictions which follow from classical relaxation theories, the magnetic relaxation in ferritin occurs faster at zero field. We note that the same conclusion follows from the observation that $d M / d H$, obtained from hysteresis loops measured below $T_{B}$, presents a sharp peak at $H=0 .{ }^{20}$ It is therefore interesting to measure magnetic viscosity at different field values, which we show next. We stress that the observed behavior cannot be due, as in other previous examples ${ }^{35}$ to interactions between different particles, which have been shown to play no significant role in our experiments.

\section{Relaxation measurements}

As reported in Ref. 20, the magnetization decay is well described by a logarithmic dependence at all values of the applied field. According to Eq. (4), the slope of these curves 


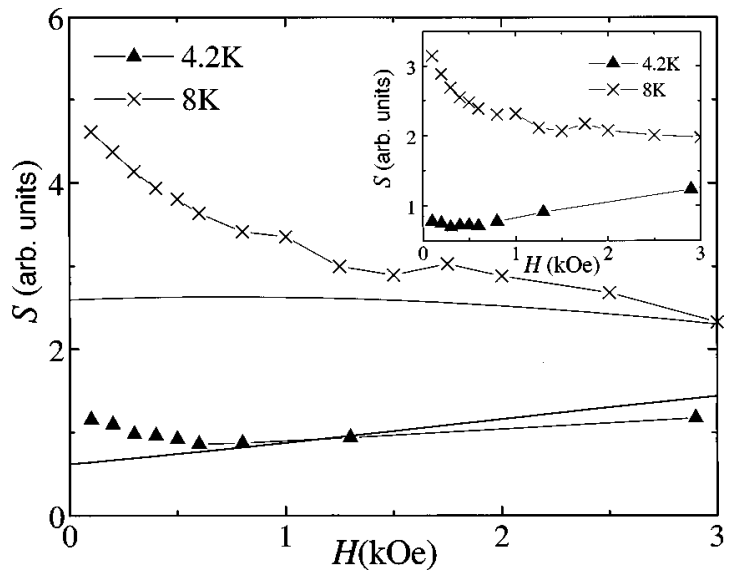

FIG. 8. Magnetic viscosity as a function of the magnetic field obtained by a new normalization method (see text) at two different temperatures. The experimental data are compared to classical predictions that follow from Eq. (5), averaged over a random distribution of easy axes (full lines). The inset shows the viscosity that was obtained previously ${ }^{20}$ by a different normalization method.

depends not only on the relaxation mechanism, but on the initial state of the sample, and the equilibrium magnetization too. It is therefore important to estimate how $\left[M_{0}\left(V_{b}\right)\right.$ $\left.-M_{e q}\left(V_{b}, H, T\right)\right]$ depends on $H$ and $T$ in order to obtain $S(T, H)$ [see Eq. (5)]. Since this magnitude cannot be directly measured, we had to use reasonable approximations.

We show next that the ac susceptibility brings the information needed in order to compare the viscosity with classical predictions. We have calculated numerically $\left[M_{0}\left(V_{b}\right)\right.$ $\left.-M_{e q}\left(V_{b}, H, T\right)\right]$, using that $V_{b} \simeq k_{B} T \ln \left(\Gamma_{0} t\right) / K_{0}$ at low fields. We take the parameters $\Gamma_{0} \simeq 10^{12} \mathrm{~s}, p \simeq 1 / 2$, and $K_{0}$ $\simeq 2.5 \times 10^{5} \mathrm{erg} / \mathrm{cm}^{3}$, which were determined previously, and fixed $t=10^{3} \mathrm{~s}$, which is approximately the center (in logarithmic scale) of our experimental time window. Data obtained with this method are shown in Fig. 8. Above $3 \mathrm{~K}, S$ decreases initially as the field increases from zero. This result suggests that magnetic relaxation is faster at zero field than at moderately large fields, in agreement with the anomalous field dependence of the ac and dc blocking temperatures, and with the peak observed in $d M / d H$ at zero field. Moreover, since the distribution of volumes is known, the viscosity obtained by using this normalization method can be compared directly to the prediction for thermally activated relaxation that follows from Eq. (5) (averaged over the random orientations of the easy axes). We see that the classical prediction reproduces (with no adjustable fitting parameters) reasonably well how the magnetic viscosity depends on the applied field above 1 kOe. However, the classical theory predicts a smooth decrease of $S$ as $H$ decreases towards zero, in contradiction with the increase observed experimentally at both temperatures.

In the inset of Fig. 8 we show viscosity data that were previously obtained by dividing $(\partial M) /[\partial \ln (t)]$ by $\left[M_{0}\right.$ $\left.-M_{e q}(H, T)\right]$, obtained experimentally for the whole sample. ${ }^{20}$ The fact that both normalization methods lead to the same result indicates that the peak observed at zero field is probably due to the field dependence of the relaxation times in the sample, and not to an improper normalization of the data, as has been suggested recently. ${ }^{22}$

\section{DISCUSSION}

We next give a simple explanation to our experimental data. The spin Hamiltonian for a ferritin molecule at zero applied field has two terms:

$$
H=H_{e x}+H_{a n} .
$$

The first term in Eq. (15) represents the exchange interaction between the atomic spins. It gives rise to a ground state with two sublattices of total spin $S_{1}$ and $S_{2}$, respectively, coupled antiferromagnetically. The second is a magnetic anisotropy term. If, as it is the case for ferritin, ${ }^{20}$ the anisotropy energy per spin is much smaller than the exchange energy this term just induces a zero-field splitting of the ground state. States $m_{S}$ and $-m_{S}$, corresponding to opposite projections of the total uncompensated spin $s=S_{1}$ $-S_{2}$ on the anisotropy axis, are nearly degenerate. Offdiagonal terms in Eq. (15) induce tunneling between these nearly degenerate spin states. Let $\Delta E_{T}$ be the tunneling splitting at maximum resonance, that is, when the longitudinal local field is equal to zero. Quantum tunneling between two nearly degenerate spin states $m_{S}$ and $-m_{S}$ is blocked if the local field fulfills $2 g \mu_{B} H_{z}^{l o c} m_{S} \gg \Delta E_{T}$. Since $\Delta E_{T}$ increases exponentially as the energy of the states increases, quantum tunneling can proceed through states, of energy $E_{m_{S}}$, which are close to, but below, the top of the classical energy barrier. $^{7-9}$ As the temperature decreases, these states are thermally depopulated and the relaxation rate decreases exponentially. Consequently, as it is observed experimentally by susceptibility measurements (see Fig. 3), more and more particles become blocked.

Coupling of the uncompensated magnetic moment to an applied magnetic field breaks the degeneracy of the spin states. At the same time, the field reduces the energy of higher excited states (with larger $s$ ) with respect to the energy of the ground state. Let's estimate the magnitude of the smallest field that induces a new crossing of spin levels. For simplicity, we neglect the field induced variation of $s$. In this simplified situation, matching of spin levels $m_{S}$ and $m_{S}^{\prime}$, localized on opposite sides of the energy barrier, occurs approximately at a longitudinal field $H_{z}=H_{1}$, given by the following expression:

$$
H_{1}=\frac{H_{a}}{2 s} .
$$

Using the average diameter of ferritin cores, it follows that $S_{1}$ and $S_{2}$ are of the order of $10^{4}$, which is much larger than the uncompensated spin $s \sim 100$, deduced from susceptibility data. Consequently, $H_{1}$ in ferritin is of the order of the anisotropy field for each sublattice $\left[\simeq\left(s / 2 S_{1}\right) H_{a}\right]$, that is, its value is of about some kOe. Using the magnetization data measured at $T=40 \mathrm{~K}$ and that $\chi_{a f} \simeq 10^{-6} \mathrm{emu} / \mathrm{g}$, we roughly estimate that, in average, $s$ increases by at most a $2 \%$ from $H=0$ to $H=1 \mathrm{kOe}$. Thus Eq. (16) should give the correct order of magnitude.

As the field increases from zero, quantum tunneling through progressively higher excited levels becomes blocked, therefore the effective energy barrier increases as was observed experimentally (see Fig. 7). Thus relaxation rates of all particles (and, consequently, the magnetic viscos- 
ity) must decrease, in agreement with experimental data (see Fig. 8). When the magnetic field is so large that tunneling is blocked for all levels below the classical energy barrier, increasing further the field value just reduces the effective barrier height. Consequently, the viscosity presents a minimum at a finite field and then increases, as expected for a classical relaxation behavior. This is precisely how the experimental $S(T, H)$ data behave above $3 \mathrm{~K}$ and also explains why the classical prediction reproduces well the data of Fig. 8 at high fields but fails near zero field. We note that resonant tunneling can be observed near zero field in ferritin because (due to the discreteness of the energy-level spectrum) the next resonance should occur at a field $H_{1}$ which is not negligibly small. This and the following resonances are not observed experimentally since, as follows from Eq. (16), the crossing field depends on the value of the uncompensated magnetic moment that is different for each molecule.

We discuss now, using the information obtained from the ac susceptibility, the viscosity data obtained below $3 \mathrm{~K}$. It was observed that $S$ increases monotonically as the applied field increases from zero ${ }^{20}$ and that it becomes temperature independent below $T_{Q} \simeq 2.4 \mathrm{~K} .{ }^{16}$ It is tempting to attribute this behavior to the existence of quantum tunneling from the lowest energy eigenstates. However, a plateau in $S(T)$ can also be explained, on classical terms, if the distribution of energy barriers increases as $1 /(K V)$ in the neighborhood of $K V_{b}\left(T_{Q}\right),{ }^{29}$ that is, if the number of particles contributing to the viscosity increases quickly as we decrease the temperature. Since relaxation experiments last for 100-4000 s, $K V_{b}\left(T_{Q}\right)$ ranges from $97-107 \mathrm{~K}$, approximately. It is clear from Fig. 4 that $f$ is a smooth decreasing function of $K V$ near this energy interval. The same should apply for low fields. Thus the observed temperature independent viscosity ${ }^{16}$ is most probably due to quantum tunneling from the ground state [see Eqs. (1) and (4)].

Now, for any reasonably large off-diagonal term in Eq. (15), $\Delta E_{T}$ for the ground state should be many orders of magnitude smaller than the Zeeman energy induced by the smallest measuring field. Thus coherent tunneling from the ground state is blocked and it must be assisted by phonons which conserve energy. ${ }^{36}$ The field dependence of $S$ that is observed experimentally follows from the fact that this tunneling mechanism is not resonant, that is, its probability is minimum at zero field but increases as $H^{3}$ approximately for each particle. It also explains that $\chi^{\prime \prime}$ does not show any indication of quantum superparamagnetism, because $T_{Q}$ at low (but large enough to block coherent tunneling) fields must be below our lowest experimentally accessible temperature.

\section{CONCLUSIONS}

We have presented a detailed experimental study of natural ferritin. ac susceptibility experiments show that dipolar magnetic interactions are negligible. Therefore we have used these data to obtain the distribution of activation volumes in our samples. Moreover, we have found that dc and ac experimental data are compatible with magnetic disorder extending to the whole ferritin core, and not only to the surface. The average diameter obtained from our results is in good agreement with the value observed by TEM.

This careful characterization of the samples is useful in order to interpret susceptibility and magnetic relaxation experiments performed at different field values. It is clear from our data that the relaxation mechanism that dominates above $3 \mathrm{~K}$ becomes slower as the field increases from zero. This result is independent of the method we use to normalize magnetic relaxation data. We therefore conclude that our data provide strong evidence on the existence at zero field of resonant tunneling between excited spin states in ferritin. Below $3 \mathrm{~K}$, thermal population of spin states where tunneling is not blocked is so small that incoherent tunneling from the lowest-lying states takes place.

J.T. and J.M.H. acknowledge support from the CICYT Project No. IN96-0027 and the CIRIT Project No. 1996PIRB-00050. F.L. and J.B. acknowledge Grant No. MAT96/448 from CICYT. The authors are grateful to the Deparment of Biochemistry (University of Zaragoza) for assistance during the preparation of the samples.

${ }^{1} \mathrm{~A}$ recent revision on the subject is found in E. M. Chudnovsky and J. Tejada, Macroscopic Quantum Tunneling of Magnetic Moment (Cambridge University Press, Cambridge, England, 1998).

${ }^{2}$ J. R. Friedman, M. P. Sarachik, J. Tejada, and R. Ziolo, Phys. Rev. Lett. 76, 3830 (1996).

${ }^{3}$ J. M. Hernandez, X. X. Zhang, F. Luis, J. Bartolome, J. Tejada, and R. Ziolo, Europhys. Lett. 35, 301 (1996).

${ }^{4}$ L. Thomas, F. Lionti, R. Ballou, D. Gatteschi, R. Sessoli, and B. Barbara, Nature (London) 383, 145 (1996).

${ }^{5}$ F. Luis, J. Bartolomé, J. F. Fernández, J. Tejada, J. M. Hernández, X. X. Zhang, and R. Ziolo, Phys. Rev. B 55, 11448 (1997).

${ }^{6}$ J. M. Hernández, X. X. Zhang, F. Luis, J. Tejada, J. R. Friedman, M. P. Sarachik, and R. Ziolo, Phys. Rev. B 55, 5858 (1997).

${ }^{7}$ D. A. Garanin and E. M. Chudnovsky, Phys. Rev. B 56, 11102 (1997).

${ }^{8}$ F. Luis, J. F. Bartolomé, and J. Fernández, Phys. Rev. B 57, 505 (1998).

${ }^{9}$ A. Fort, A. Rettori, J. Villain, D. Gatteschi, and R. Sessoli, Phys. Rev. Lett. 80, 612 (1998).

${ }^{10}$ L. Néel, J. Phys. Soc. Jpn. 17, 676 (1962).

${ }^{11}$ B. Barbara and E. M. Chudnovsky, Phys. Lett. A 145, 205 (1990).

${ }^{12}$ W. H. Massover, Micron 24, 389 (1993).

${ }^{13}$ K. M. Towe and W. F. Bradley, J. Colloid Interface Sci. 24, 384 (1967).

${ }^{14}$ E. P. Bauminger and W. F. Bradley, Hyperfine Interact. 50, 489 (1989).

${ }^{15}$ D. P. E. Dickson, N. M. K. Reid, C. Hunt, H. D. Williams, M. El-Hilo, and K. O'Grady, J. Magn. Magn. Mater. 125, 345 (1993).

${ }^{16}$ J. Tejada and X. X. Zhang, J. Phys.: Condens. Matter 6, 263 (1994). 
${ }^{17}$ M-E. Y. Mohie-Eldin, R. B. Frankel, and L. Gunther, J. Magn. Magn. Mater. 135, 65 (1994).

${ }^{18}$ S. H. Kilcoyne and R. Cywinski, J. Magn. Magn. Mater. 140-144, 1466 (1995).

${ }^{19}$ S. A. Makhlouf, F. T. Parker, and A. E. Berkowitz, Phys. Rev. B 55, R14 717 (1997).

${ }^{20}$ J. Tejada, X. X. Zhang, E. del Barco, J. M. Hernández, and E. M. Chudnovsky, Phys. Rev. Lett. 79, 1754 (1997).

${ }^{21}$ J. R. Friedman, U. Voskoboynik, and M. P. Sarachik, Phys. Rev. B 56, 10793 (1997).

${ }^{22}$ R. Sappey, E. Vincent, N. Hadacek, F. Chaput, J. P. Boilot, and D. Zins, Phys. Rev. B 56, 14551 (1997).

${ }^{23}$ D. D. Awschalom, J. F. Smyth, G. Grinstein, D. P. DiVincenzo, and D. Loss, Phys. Rev. Lett. 68, 3092 (1992).

${ }^{24}$ S. Gider, D. D. Awschalom, T. Douglas, K. Wong, S. Mann, and G. Cain, J. Appl. Phys. 79, 5324 (1996).

${ }^{25}$ M. Hanson, C. Johansson, and S. Morup, Phys. Rev. Lett. 81, 735 (1998).

${ }^{26}$ H. Pfeiffer, Phys. Status Solidi A 118, 295 (1990).

${ }^{27}$ J. Tejada, X. X. Zhang, and E. M. Chudnovsky, Phys. Rev. B 47,
14977 (1993).

${ }^{28}$ E. Vincent, J. Hamman, P. Prené, and E. Tronc, J. Phys. I 4, 273 (1994).

${ }^{29}$ B. Barbara and L. Gunther, J. Magn. Magn. Mater. 128, 35 (1993).

${ }^{30}$ M. I. Shliomis and V. I. Stepanov, J. Magn. Magn. Mater. 122, 176 (1993).

${ }^{31}$ L. Lundgren, P. Svedlindh, and O. Beckman, J. Magn. Magn. Mater. 25, 33 (1981).

${ }^{32}$ F. Luis, J. Bartolomé, J. Tejada, and E. Martínez, J. Magn. Magn. Mater. 157-158, 266 (1996).

${ }^{33}$ M. Hanson, C. Johanson, and S. Morup, J. Phys.: Condens. Matter 5, 725 (1993).

${ }^{34}$ M. Hanson, C. Johanson, and S. Morup, J. Phys.: Condens. Matter 7, 9263 (1993).

${ }^{35}$ W. Luo, S. R. Nagel, T. F. Rosenbaum, and R. E. Rosensweig, Phys. Rev. Lett. 67, 2721 (1991).

${ }^{36}$ P. Politi, A. Rettori, F. Hartmann-Boutron, and J. Villain, Phys. Rev. Lett. 75, 537 (1996). 